\title{
Identification of MMP9 as a novel key gene in mantle cell lymphoma based on bioinformatic analysis and design of cyclic peptides as MMP9 inhibitors based on molecular docking
}

\author{
WEI YAN ${ }^{1}$, SHAWN XIANG LI $^{2}$, MINJIE WEI ${ }^{1}$ and HUA GAO ${ }^{1}$ \\ ${ }^{1}$ Department of Pharmacology, School of Pharmacy; ${ }^{2}$ International College, \\ China Medical University, Shenyang, Liaoning 110122, P.R. China
}

Received February 12, 2018; Accepted September 3, 2018

DOI: $10.3892 / o r .2018 .6682$

\begin{abstract}
Mantle cell lymphoma (MCL) is an aggressive disease. MCL is associated with poor patient prognosis and limited survival. To identify key genes and explore targeting cyclic peptide inhibitors for the treatment of MCL, we downloaded two gene expression profiles (GSE32018 and GSE9327) from the Gene Expression Omnibus (GEO) database. We screened 84 differentially expressed genes (DEGs). Pathway analysis showed that DEMs were mainly enriched in the 'Pathway in cancer', 'PI3K-Akt signaling pathway', 'Cytokine-cytokine receptor interaction', 'Rap1 signaling pathway', 'NF-кB signaling pathway' and 'Leukocyte trans-endothelial migration'. We subsequently constructed a protein-protein interaction (PPI) network of DEGs. In addition, matrix metalloproteinase 9 (MMP9) with a high degree in the PPI network was identified as a hub gene in MCL. Meanwhile in the Molecular Complex Detection (MCODE) analysis, MMP9 was located in the important cluster. Thus, MMP9 can be used as a therapeutic target for MCL and we designed cyclic peptides as MMP9 inhibitors. MMP9 protein structure was gathered from the Protein Data Bank (PDB),
\end{abstract}

Correspondence to: Dr Hua Gao, Department of Pharmacology, China Medical University, 77 Puhe Road, Shenyang, Liaoning 110122, P.R. China

E-mail: cmu-gaohua@hotmail.com

Abbreviations: MCL, mantle cell lymphoma; GEO, Gene Expression Omnibus; DEGs, differentially expressed genes; PPI, protein-protein interaction; MCODE, Molecular Complex Detection; PDB, Protein Data Bank; MOE, Molecular Operating Environment; R-CHOP, rituximab, cyclophosphamide, doxorubicin hydrochloride, vincristine, prednisone; MMP9, matrix metalloproteinase 9; MMPs, matrix metalloproteinases; MMPIs, MMP inhibitors; GO, Gene Ontology; KEGG, Kyoto Encyclopedia of Genes and Genomes; 3D, three-dimensional; FnII, fibronectin type II; BP, biological process; $\mathrm{CC}$, cell component; MF, molecular function; ECM, extracellular matrix

Key words: MMP9, mantle cell lymphoma, protein-protein interaction, cyclic peptides, inhibitors, molecular docking with a PDB ID: 1L6J. MMP9 and cyclic peptides were docked using Molecular Operating Environment (MOE) software after structural optimization. It was revealed that cyclic peptide 2 bound deeply in the binding pocket of MMP9 and had interaction with the active-site $\mathrm{Zn}^{2+}$ ion in the catalytic domain. Cyclic peptides 1, 2, 4-6 also displayed potential interaction with active residues of MMP9; thus, these cyclic peptides can serve as potential drug candidates to block MMP9 activity and future studies are warranted to confirm their efficacy.

\section{Introduction}

Mantle cell lymphoma (MCL) is a type of non-Hodgkin's lymphoma (NHL) and is molecularly identified by chromosomal translocation $\mathrm{t}(11 ; 14)(\mathrm{q} 13 ; \mathrm{q} 32)$. This disease is characterized by quick relapses plus poor outcomes over the long-term, predominantly affecting older adults (1). The clinical course of MCL is mostly invasive, and most cases have extensive extranodal infiltration and bone marrow invasion. In younger patients, randomized trials have established the advantage of dose-intensified, cytarabine-containing induction with or without transplantation of stem cells. In elderly patients, treatment with R-CHOP (rituximab, doxorubicin hydrochloride, cyclophosphamide, vincristine and prednisone) has greatly prolonged overall survival (2). Yet, the great majority of patients unfortunately will suffer relapse. Therefore, the pathogenesis of MCL and effective therapeutic drugs for this disease are still under active exploration.

Microarray technology is widely utilized for the study of general genetic aberrations in MCL (3). Nevertheless, there are studies integrating these microarray datasets to identify key genes in MCL. Herein, we analyzed two sizeable and representative gene expression profiles to identify differentially expressed genes (DEGs) between MCL and normal lymph gland. In addition, functional enrichment analysis and protein-protein interaction (PPI) analysis were performed for the DEGs, to uncover key genes in MCL. We found that matrix metalloproteinase 9 (MMP9) plays a vital role in these key genes.

Invasion and metastasis are important biological characteristics of malignant tumors including MCL. Tumor growth, 
invasion and metastasis involve a complex multistep process, including the division and proliferation of tumor cells, the degradation of the extracellular matrix, cell escape through the basement membrane, migration into the blood circulation system, tumor cell growth at secondary sites and a series of activities, at the same time accompanied by the formation of new blood vessels (4). Studies have shown that MMP9, a member of the matrix metalloproteinase (MMP) family is highly expressed in various tumors and plays a major role in the growth, metastasis and angiogenesis of primary and secondary tumors (5-8).

MMPs inhibitors (MMPIs) have been a major focus of research in recent years. Since the 1990s, the world's major pharmaceutical companies have invested heavily in the research and development of MMPIs. At present, more than a dozen MMPIs have entered the clinical stage and there are also a large number of compounds that are undergoing pre-clinical research. These chemically synthesized MMPIs can be broadly classified into peptide MMPIs, non-peptide MMPIs, tetracycline MMPIs and bisphosphonate MMPIs (9). As a result, MMPIs are of great significance as a new antitumor agent. Moreover, peptide drugs have low adverse reactions, low immunogenicity, and are easy to synthesize and transform, which makes these agents worthy of further research.

The affinity of the molecules against their target proteins were generated with computational techniques. Molecular docking is a method of binding orientation prediction of molecules with protein targets (10). Therefore, molecular docking is regarded as a vital technique in drug design. The aim of the present study was to screen new cyclic peptides as activated MMP9 inhibitors with which to target hydrophobic pockets of MMP9 by using computational docking techniques.

\section{Materials and methods}

Microarray data. Two sizeable gene expression profiles including lymph nodes of MCL and normal lymph nodes (GSE32018 and GSE9327) $(11,12)$ were acquired from the GEO (Gene Expression Omnibus) (http://www.ncbi.nlm.nih. gov/geo). The GEO database is a functional genomics data repository. It stores microarray and sequencing data. The two gene expression profiles included $62 \mathrm{MCL}$ samples and 14 normal lymph node samples.

Data processing. GEO2R (http://www.ncbi.nlm.nih. gov/geo/geo2r/) is a program that allows the investigator to compare two or more groups of samples within a GEO dataset. This is performed to identify DEGs across experimental circumstances. GEO2R analyzes original submitter-supplied processed microarray data using the GEO query and limma R packages (http://www.bioconductor. org/packages/release/bioc/html/limma.html) from the Bioconductor project. In the present study, GEO2R was applied to obtain DEGs in MCL compared with normal lymph nodes. The false-positive results for the microarray were then corrected by adjusted P-value (adj. P-value) using Benjamini-Hochberg method. The cut-off criterion was set as $\mathrm{P}<0.05$ and ' $\log \mathrm{FCl}>1$ '.

Gene Ontology (GO) and Pathway Analysis. The Database for Annotation, Visualization and Integrated Discovery (DAVID; http://david.abcc.ncifcrf.gov/) is a website that offers the interpretation of genes. For our study, the DAVID database was used to perform pathway analysis. Gene Ontology holds three hierarchies: Cellular component (CC), Biological process (BP) and Molecular function (MF) (13).

Pathway Analysis is a functional analysis that can map genes to KEGG pathways (13). This study performed GO analysis and KEGG pathway analysis using only overlapped DEGs in the two independent datasets (GSE32018 and GSE9327). The significance of the GO and pathway term enrichment in the DEGs are denoted by P-value. The cut-off point was set as $\mathrm{P}<0.05$.

Establishment of PPI network. STRING (http://string-db.org) was utilized to identify interaction networks of protein products of these DEGs. The cut-off criterion was set as 'Confidence score $\geq 0.7$ '. The Cytoscape 3.5.1 software (Institute of Systems Biology, Seattle, WA, USA) was used to build protein-protein interaction (PPI) networks among the DEGs in MCL. The hub genes were identified by Cytohubba plugin (14), and the top 10 nodes were selected as ranked by degree. Molecular Complex Detection (MCODE) (15) was applied in order to find clusters of genes in the PPI network.

Receptor refinement. Cyclic peptides were docked against activated MMP9 by the Molecular Operating Environment (MOE) software 2016.08 (Chemical Computing Group ULC, Montreal, Quebec, Canada) (http://www.chemcomp.com/). The 3D structure of MMP9 was obtained from the database of the Protein Data Bank (PDB) (https://www.rcsb.org/) using PDB ID:1L6J. Optimization of the structure occurred by adding hydrogen using software. To optimize the structure, $\mathrm{H}_{2} \mathrm{O}$ molecules were detached from the structure and 3D protonation was conducted to change the state into the ionization level. Moreover, energy minimization was performed using certain parameters. Docking studies used this minimized structure.

Design of the peptide. MMP9 usually exists as proMMP9, and the construction of proMMP9 includes the propeptide, the catalytic domain plus three fibronectin type II (FnII) domains (Fig. 1) (16). ProMMP9 is triggered by autoproteolytic cleavage of the propeptide, in vivo or in vitro, by reacting with an organomercurial compound or other proteases (17). The stretches of propeptide residues 97-100 that are inserted into the active-site cleft, block access to the catalytic zinc. Cleavage of the propeptide opens up the active site, facilitating interactions with substrate or inhibitors. According to the study on the propeptide, we selected amino acid sequence TPRCGVPDL from the propeptide including key amino acid residues 97-100 PRCG as the template peptide. Non-polar aromatic amino acid (F/Y/W) was added in key amino acids, and there were two alternatives: replacement of the first position amino acid $\mathrm{P}$ or the fourth position $\mathrm{G}$. The second position of key amino acid residues had a likeness of interaction with polar positive charged amino acid residue $\mathrm{K}$, whereas the preference for the third position according to the amino acid properties was polar amino acids (R/N/D/Q/E/H/C/S/T) (Fig. 2). Six peptides were designed to investigate their potential against active MMP9. Furthermore, disulfide bridges were created by adding cysteine 


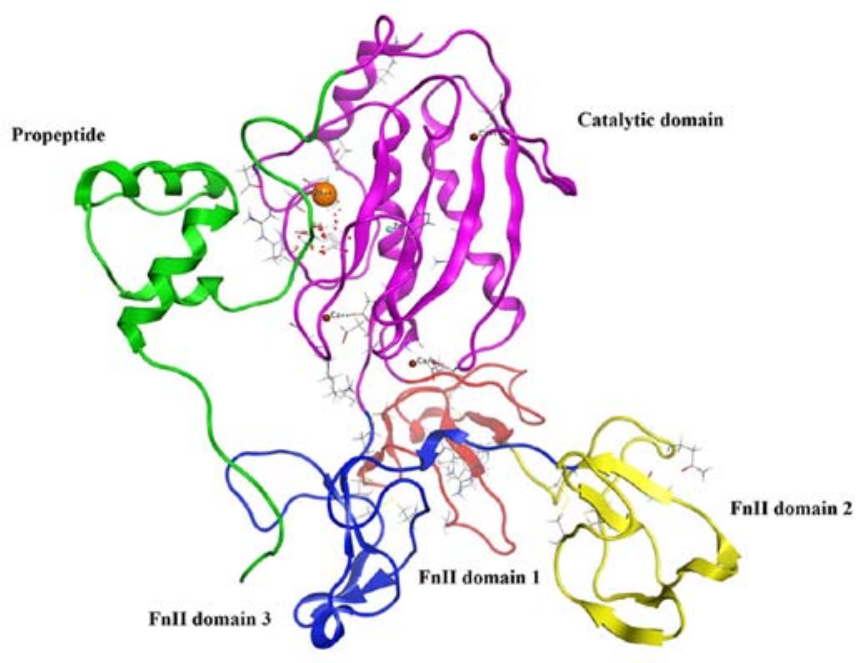

Figure 1. Ribbon drawing of proMMP9. The propeptide is indicated in green. The catalytic domain is shown in pink and three FnII domains are in red, yellow and blue. $\mathrm{Ca}$ atoms are shown as red spheres and $\mathrm{Zn}$ atom as a large orange sphere in the active site. Residues of hydrophobic zone S2, S1', S1' and S2' are shown as red and white small spheres.

residues at both ends in order to convert these peptides into more stable cyclic peptides. ACD/ChemSketch software 12.0 (ACD/Labs, Toronto, Ontario, Canada) (www.acdlabs.com) was used to convert the designed peptides into their respective 3D structures.

Peptide refinement. All of the peptides were optimized. The MOE software was used to achieve this task, by adding hydrogen. Energy of the peptides was minimized using parameters such as gradient: 0.05, Force Field: MMFF94X, Chiral Constraint and Current Geometry. In addition, Conformational Search of these peptides was conducted by the LowModeMD method, and the results were saved in mdb database for further docking studies.

Molecular docking. Docking of these peptides with the active MMP9 was conducted with the algorithm of the MOE software. Parameters were set as Re-scoring function, London dG; placement, Triangle matcher; Retain, 5; Refinement, Force Field; and Re-scoring 2, London dG. The docking program of MOE provides the correct conformation (with the rotation

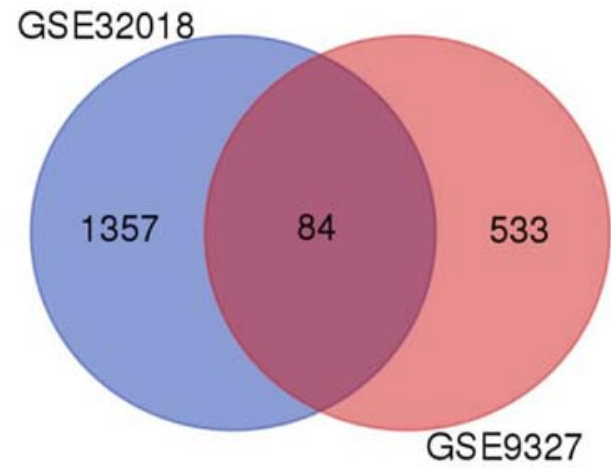

Figure 3. Identification of differentially expressed genes in mRNA expression profiling datasets GSE32018 and GSE9327.

of bonds, structure of the molecule is not rigid) of the ligand. $\mathrm{S}$ score was the basis for the choice of top conformation for each peptide. The top conformations also received further evaluation in order to study the hydrogen bonding $/ \pi-\pi$ interactions.

\section{Results}

Identification of DEGs in MCL. GEO2R analysis demonstrated that a total of 1,441 and 617 DEGs were acquired in the GSE32018 and GSE9327 datasets, respectively. Moreover, 84 DEGs were also identified in both datasets (Fig. 3).

Functional enrichment analysis. GO analysis illustrated that the most significantly enriched GO terms corresponded to DEGs were 'Signal transduction' (GO: BP) (Fig. 4A), 'Extracellular exosome' (GO: CC) (Fig. 4B) and 'Protein binding' (GO: MF) (Fig. 4C).

Additionally, KEGG pathway analysis illustrated that the DEGs were enriched in 24 pathways, such as 'Pathway in cancer', 'PI3K-Akt signaling pathway', 'Cytokine-cytokine receptor interaction', 'Rap1 signaling pathway', 'NF- $\mathrm{B}$ signaling pathway' and 'Leukocyte trans-endothelial migration' (Table I).

Establishment of the PPI network and identification of MMP9 as a hub gene. The PPI network of DEGs was constructed by STRING; it consisted of 52 nodes and 95 edges (Fig. 5A).

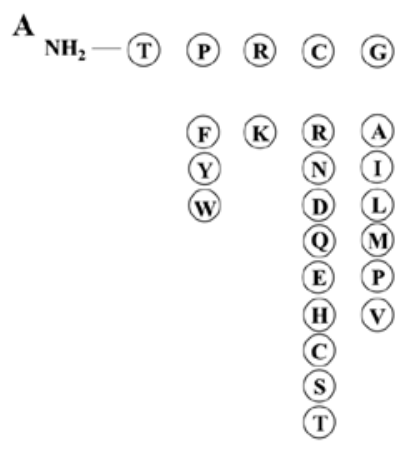

B $\mathrm{NH}_{2}$ - (T) (P) (B) (G) (V)(D)(L) $-\mathrm{COOH}$

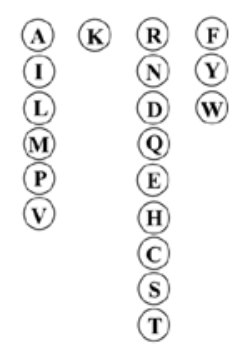

Figure 2. Alternative proposals for designing the peptides. (A) Non-polar aromatic amino acids (F/Y/W) replace the first position amino acid P. (B) Non-polar aromatic amino acids $(\mathrm{F} / \mathrm{Y} / \mathrm{W})$ replace the fourth position $\mathrm{G}$. 

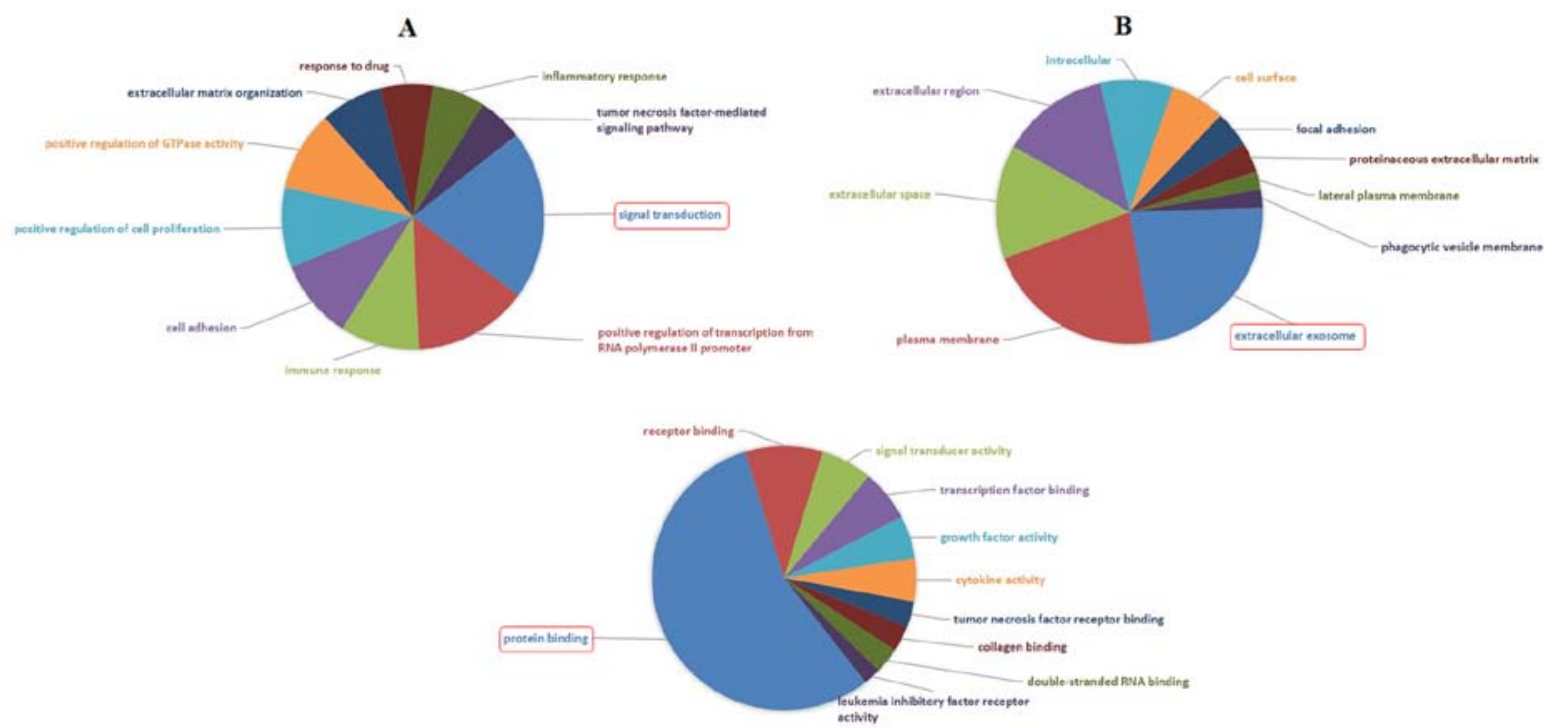

C

Figure 4. Enriched Gene ontology (GO) terms for differentially expressed genes: (A) biological process (BP); (B) cellular component (CC); (C) molecular function (MF). The red boxes represent the most significantly enriched GO terms: 'Signal transduction', 'Extracellular exosome' and 'Protein binding'.

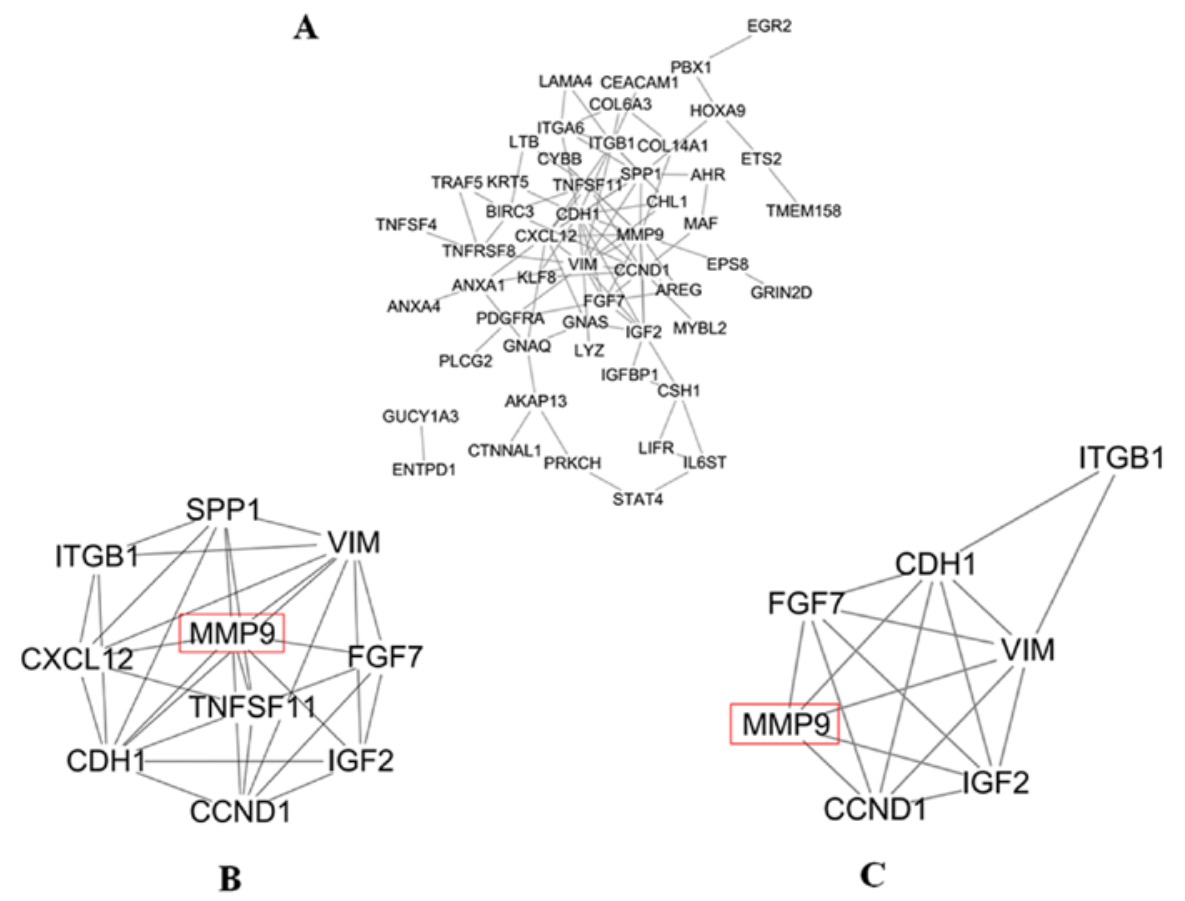

Figure 5. Identification of MMP9 as a hub gene. (A) Protein-protein interaction network of differentially expressed genes. (B) The top 10 degree genes in the protein-protein interaction network. (C) The most significant cluster selected by MCODE analysis. MMP9, matrix metalloproteinase 9.

Moreover, the top 10 degree genes in the PPI network were selected in MCL using cytohubba, e.g. VIM, MMP9, CDH1, CCND1, ITGB1, SPP1, CXCL12, IGF2, TNFSF11 and FGF7 genes (Fig. 5B). In addition, 4 genes in the top 10 degree genes were identified as hub genes in MCL, e.g. VIM, MMP9, CDHI and $C C N D 1$ genes, when 'Degree $\geq 10$ ' was set as the cut-off criterion. Then, using MCODE, clusters were selected from the PPI network. It was shown that the most significant cluster had 7 nodes and 17 edges. MCODE analysis demonstrated that the most significant cluster contained the four hub genes (VIM, MMP9, CDH1 and CCND1) (Fig. 5C). A member of the cancer-related genes, MMP9 had the second highest degree in the PPI network of MCL. Its involvement in invasion and metastasis was found to be consistent with the malignant biological behavior of MCL. Meanwhile, the 3D X-ray crystallography structure of MMP9 was retrieved from the database of PDB using PDB ID:1L6J which had a resolution of $2.5 \AA$. Therefore, MMP9 was chosen for follow-up study.

MMP9 serves a key function in lymphoma progression. The association between MMP9 and lymphoma was further analyzed. Using Oncomine analysis (18), the expression level 
Table I. Enriched pathways corresponding to differentially expressed genes (DEGs).

\begin{tabular}{|c|c|c|}
\hline Term & Count & Genes \\
\hline Pathways in cancer & 14 & $\begin{array}{l}\text { FGF7, MMP9, CDH1, BIRC3, CXCL12, ITGB1, LAMA4, CCND1, } \\
\text { ITGA6, GNAQ, PLCG2, PDGFRA, GNAS, TRAF5 }\end{array}$ \\
\hline PI3K-Akt signaling pathway & 9 & $\begin{array}{l}\text { CSH1, LAMA4, CCND1, FGF7, ITGA6, COL6A3, PDGFRA, ITGB1, } \\
\text { SPP1 }\end{array}$ \\
\hline Cytokine-cytokine receptor interaction & 8 & TNFSF4, TNFSF11, IL6ST, PDGFRA, LIFR, TNFRSF8, CXCL12, LTB \\
\hline Focal adhesion & 8 & LAMA4, CCND1, ITGA6, COL6A3, PDGFRA, BIRC3, ITGB1, SPP1 \\
\hline Rap1 signaling pathway & 7 & $F Y B, F G F 7, G N A Q, P D G F R A, C D H 1, G N A S, I T G B 1$ \\
\hline HTLV-I infection & 7 & CCND1, EGR2, ETS2, PDGFRA, HLA-A, HLA-DMB, MYBL2 \\
\hline $\mathrm{NF}-\kappa \mathrm{B}$ signaling pathway & 6 & TNFSF11, PLCG2, BIRC3, CXCL12, TRAF5, LTB \\
\hline Small cell lung cancer & 6 & LAMA4, CCND1, ITGA6, BIRC3, TRAF5, ITGB1 \\
\hline Leukocyte transendothelial migration & 5 & $C Y B B, M M P 9, P L C G 2, C X C L 12, I T G B 1$ \\
\hline ECM-receptor interaction & 5 & LAMA4, ITGA6, COL6A3, ITGB1, SPP1 \\
\hline Toxoplasmosis & 5 & LAMA4, ITGA6, HLA-DMB, BIRC3, ITGBI \\
\hline Platelet activation & 5 & GNAQ, PLCG2, GUCY1A3, GNAS, ITGB1 \\
\hline Cell adhesion molecules (CAMs) & 5 & LAMA4, ITGA6, COL6A3, ITGB1, SPP1 \\
\hline Jak-STAT signaling pathway & 5 & LAMA4, ITGA6, HLA-DMB, BIRC3, ITGB1 \\
\hline Calcium signaling pathway & 5 & GNAQ, GRIN2D, PLCG2, PDGFRA, GNAS \\
\hline Epstein-Barr virus infection & 5 & PLCG2, VIM, HLA-A, ENTPD1, TRAF5 \\
\hline Inflammatory bowel disease (IBD) & 4 & $M A F, S T A T 4, R O R A, H L A-D M B$ \\
\hline Melanoma & 4 & $C C N D 1, F G F 7, P D G F R A, C D H 1$ \\
\hline Salivary secretion & 4 & GNAQ, LYZ, GUCY1A3, GNAS \\
\hline Gap junction & 4 & GNAQ, PDGFRA, GUCY1A3, GNAS \\
\hline Rheumatoid arthritis & 4 & TNFSF11, HLA-DMB, CXCL12, LTB \\
\hline Circadian entrainment & 4 & GNAQ, GRIN2D, GUCY1A3, GNAS \\
\hline $\begin{array}{l}\text { Inflammatory mediator regulation of } \\
\text { TRP channels }\end{array}$ & 4 & GNAQ, PLCG2, PRKCH, GNAS \\
\hline Bladder cancer & 3 & $C C N D 1, M M P 9, C D H 1$ \\
\hline
\end{tabular}

Table II. Peptide interaction with MMP9.

\begin{tabular}{|c|c|c|c|c|c|}
\hline Sr. No. & Peptide & S score & RMSD & Interacting residues & Close contact residues \\
\hline Template & Cys-TPRCGVPDL-Cys & -61.8677 & 6.2413 & $\begin{array}{l}\text { Zn500, Leu } 188, \\
\text { Glu402, His411 }\end{array}$ & $\begin{array}{l}\text { Leu187, Ala189, His190, Ala191, Phe192, } \\
\text { His401, His405, Asp410, Pro421 }\end{array}$ \\
\hline 1 & Cys-TFKEGVPDL-Cys & -62.0522 & 8.4138 & Zn500 & $\begin{array}{l}\text { Gly } 186, \text { Leu } 187 \text {, His } 190, \text { His } 401, \text { His } 405 \text {, } \\
\text { His411, Pro421, Met422 }\end{array}$ \\
\hline 2 & Cys-TFKEIVPDL-Cys & -85.8988 & 8.1607 & $\begin{array}{l}\text { Zn500, His } 190, \\
\text { Glu402 }\end{array}$ & $\begin{array}{l}\text { Leu187, Ala189, His } 401, \text { His } 405, \text { His } 411, \\
\text { Pro421, Met } 422\end{array}$ \\
\hline 3 & Cys-TFKEMVPDL-Cys & -82.7207 & 4.0789 & $\mathrm{Zn} 500$ & $\begin{array}{l}\text { Gly186, Leu187, Leu188, Ala191, } \\
\text { His401, Glu402, His405, His411, Pro421, } \\
\text { Met422, Tyr423 }\end{array}$ \\
\hline 4 & Cys-TFKELVPDL-Cys & -68.4973 & 3.3641 & Zn500 & $\begin{array}{l}\text { Gly 186, Leu187, Leu188, His401, } \\
\text { Glu402, His405, Asp410, His411, Pro421, } \\
\text { Met422, Tyr423 }\end{array}$ \\
\hline 5 & Cys-TFKRGVPDL-Cys & -71.9705 & 1.6998 & $\begin{array}{l}\text { Zn500, Ala191, } \\
\text { Leu409, Asp410 }\end{array}$ & $\begin{array}{l}\text { Leu187, Phe192, Pro193, His401, His405, } \\
\text { Leu409, Asp410, His411, Pro421 }\end{array}$ \\
\hline 6 & Cys-TPKCWVPDL-Cys & -68.3860 & 1.7706 & Zn500, Ala191 & $\begin{array}{l}\text { Leu187, Ala189, His190, Phe192, His401, } \\
\text { Glu402, His405, Asp410, His411, Pro421 }\end{array}$ \\
\hline
\end{tabular}


A

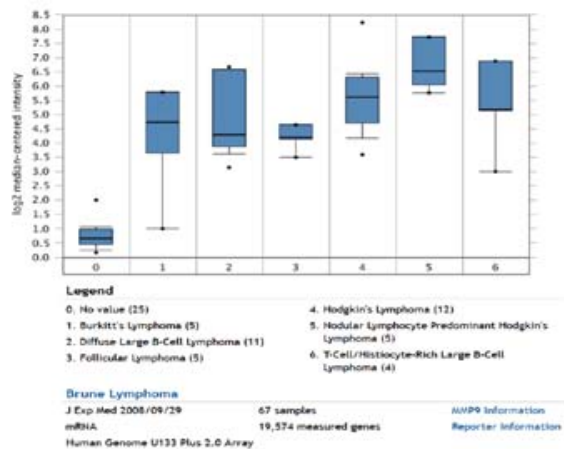

C

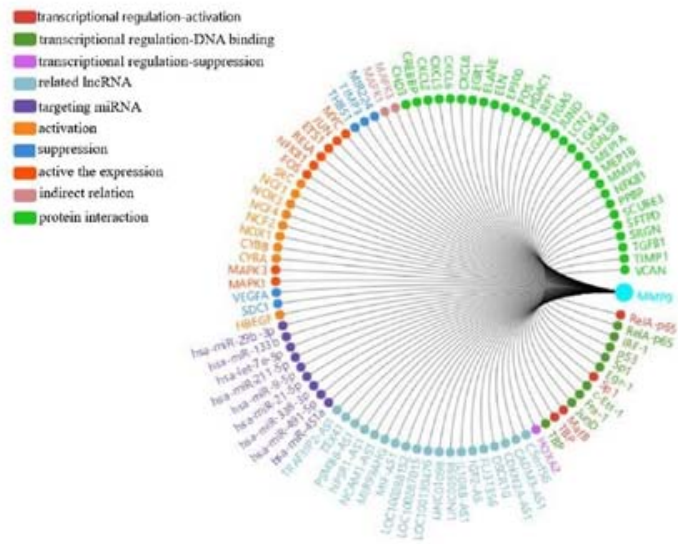

B

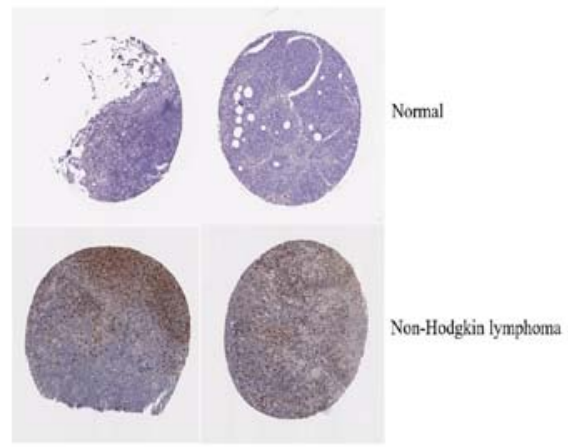

D

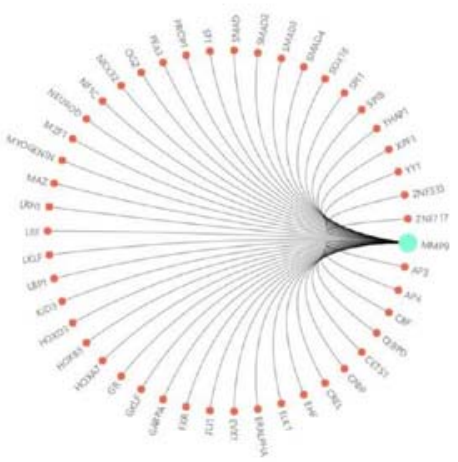

Figure 6. Expression level and regulatory network of MMP9 in a number of types of lymphoma. (A) The mRNA expression level results of MMP9 in a number of types of lymphoma were obtained from Oncomine dataset. The median, upper and lower quartiles are plotted, and the whiskers indicate the data range. The points that are $>1.5 \mathrm{x}$ the interquartile range are marked as outliers. (B) Representative immunohistochemical staining of MMP9 (CAB000348) in two samples of lymphoma and normal lymphoid tissues obtained from Human Protein Atlas. (C) The regulatory network and (D) related transcription factors of MMP9 were predicated. MMP9, matrix metalloproteinase 9.

A

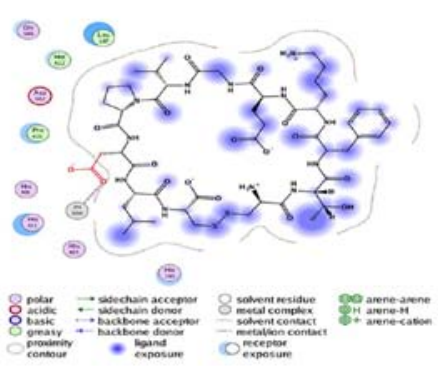

D

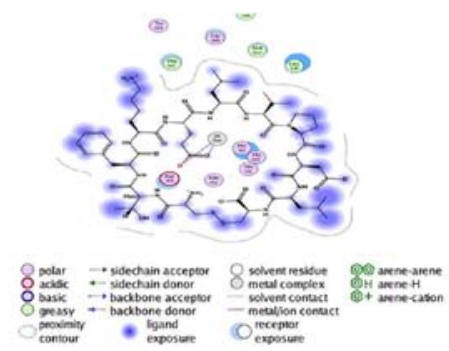

B

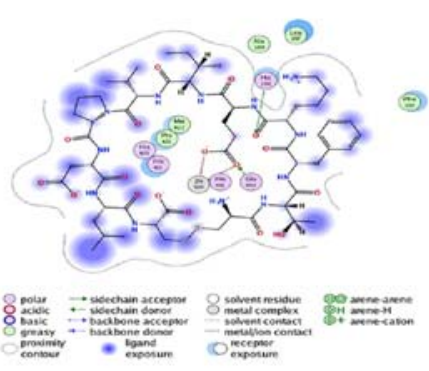

$\mathbf{E}$
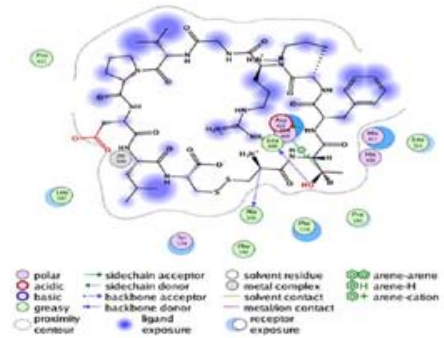

C

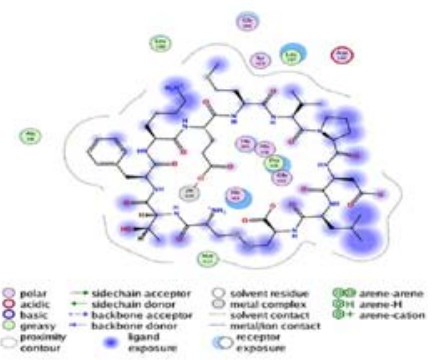

F

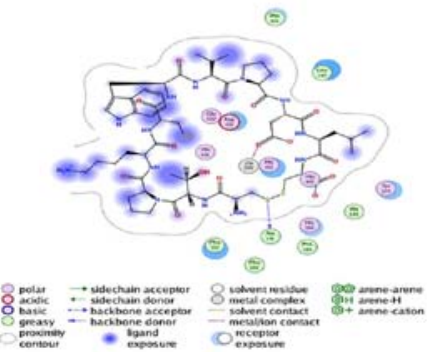

Figure 7. Binding interactions of peptides with the active residues of MMP9 presented by 2D mode. (A-F) correspond to peptide 1 to peptide 6. MMP9, matrix metalloproteinase 9 .

of MMP9 was determined in lymphoma. It was identified that MMP9 was upregulated in a number of types of lymphoma, including Burkitt's lymphoma, diffuse large B cell lymphoma, follicular lymphoma and Hodgkin's lymphoma (19) (Fig. 6A). In addition, the immunohistochemical staining results of MMP9, obtained from the Human Protein Atlas database (www. proteinatlas.org), were analyzed, which revealed that MMP9 was upregulated in non-Hodgkin lymphoma, compared with 
A

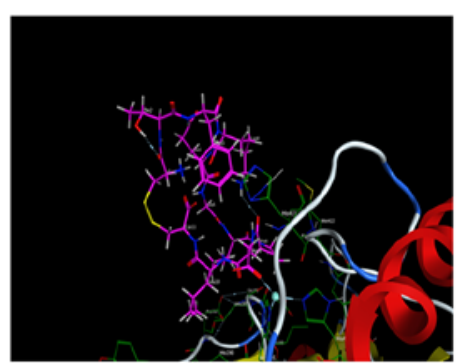

D

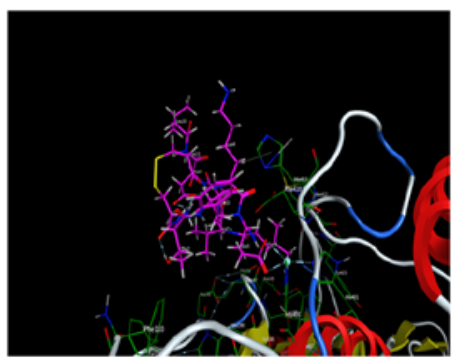

B

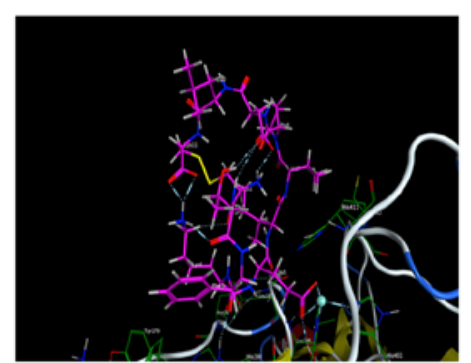

E

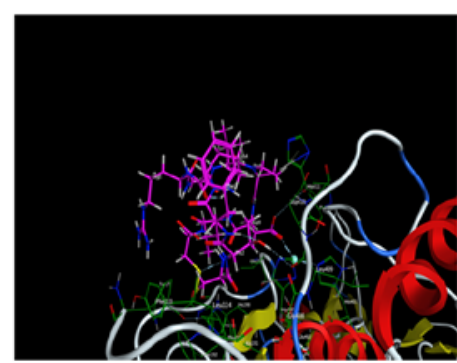

C

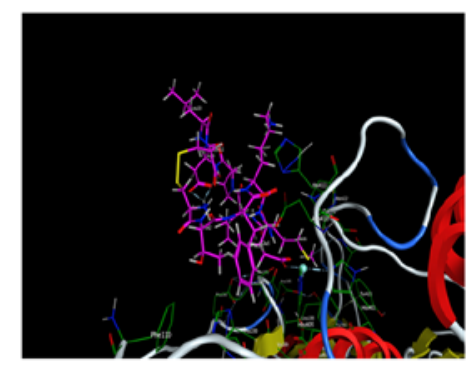

F

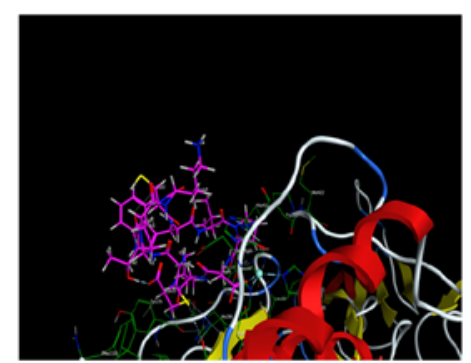

Figure 8. Binding interactions of peptides with active residues of MMP9 presented by 3D mode. (A-F) correspond to peptide 1 to peptide $6 . \mathrm{Zn}$ atoms are shown as cyan-colored spheres in the active site and designed cyclic peptides are indicated as pink skeleton structures. MMP9, matrix metalloproteinase 9.

A

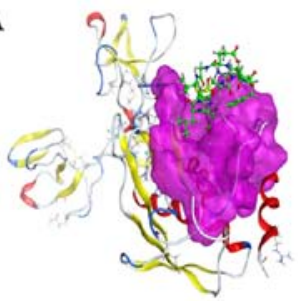

D

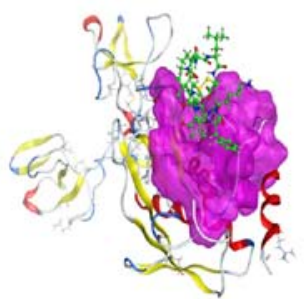

B

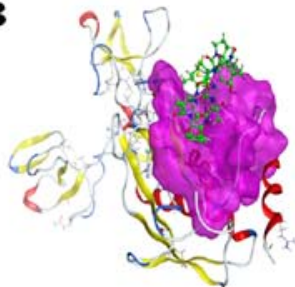

$\mathbf{E}$

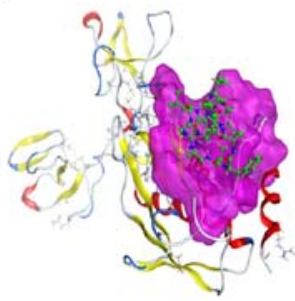

C

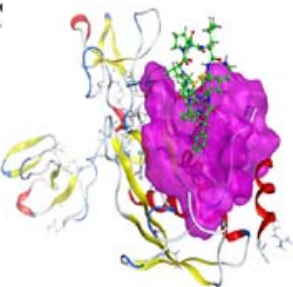

$\mathbf{F}$

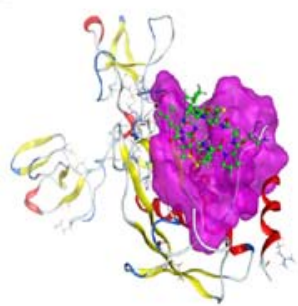

Figure 9. Docked complexes of peptides with the binding pocket of MMP9. (A-F) correspond to peptide 1 to peptide 6. Designed cyclic peptides are shown as green skeleton structures. MMP9, matrix metalloproteinase 9.

normal lymphoid tissue (Fig. 6B) (http://www.proteinatlas org/ENSG00000100985-MMP9/pathology/tissue/lymphoma). Using Gene-RADAR analysis (www.gcbi.com.cn), the regulatory network and related transcription factors of MMP9 were predicted (Fig. 6C and D). Taken together, MMP9 may be involved in lymphoma progression.

Molecular docking. MOE docking program generated 10 conformations for every piece of peptide. Conformations were sorted according to two parameters: $\mathrm{S}$ score plus top ranking conformation with minimum S score was further analyzed. Peptide 2 was ranked as the top conformation followed by peptide 3. Other conformations had scores close to each other (Table II). The most promising conformation for each peptide was analyzed to find hydrogen bonding $/ \pi-\pi$ interactions .
Interaction analysis. Six peptides were found to be able to bind to $\mathrm{Zn}^{2+}$ in the active site and affect the enzymatic activity. Peptide 2 also exhibited interactions with the two residues (His190 and Glu402), in addition to having a minimum $\mathrm{S}$ score. Therefore, peptide 2 could serve as a drug candidate against active MMP9. Additionally, peptide 5 was ranked third, and it had potential interactions with Ala191, Leu409 and Asp410 of the active sites. Peptide 6 had strong interaction with Ala191 of the active sites. All other peptides (1, 3 and 4) did not have any potential interaction with the active site but had hydrophobic contact with active residues of the catalytic domain. Interacting residues of active sites are shown in Table II. Interactions between receptor and ligands are shown in Figs. 7 and 8. The binding mode of ligands with the receptor protein is shown in Fig. 9. 


\section{Discussion}

Mantle cell lymphoma (MCL) is a rare and incurable type of non-Hodgkin's lymphoma. Standard treatment for young patients includes cytarabine-based induction, which is then followed up with autologous stem cell transplant. This is well supported by large randomized trial data. Many patients are not eligible for this intensive approach due to advanced age, comorbidities and increased toxicities (20). Molecular targeting strategies have improved the outcome of MCL patients (21). These targeting strategies include bortezomib, lenalidomide, temsirolimus, and especially inhibitors of the B-cell receptor pathway. Nevertheless, the efficacy noted is not identical for all patients with MCL, partly due to the complexity of MCL. Therefore, investigation of the molecular mechanisms involved in MCL and the exploration of specifically targeted new agents are essential and urgent.

Eighty-four differentially expressed genes (DEGs) were identified in this study. The DEGs were enriched in 24 pathways, such as 'Pathway in cancer', 'PI3K-Akt signaling pathway', 'Cytokine-cytokine receptor interaction', 'Rap1 signaling pathway', 'NF-кB signaling pathway' and 'Leukocyte transendothelial migration'. Among the 84 DEGs, 4 genes were identified as hub genes in MCL: VIM, $M M P 9, C D H 1$ and $C C N D 1$ genes. Additionally, 2 clusters were obtained from the PPI network using MCODE, and the most significant cluster contained the four hub genes. Finally, tumor-related gene MMP9 attracted our attention, and the 3D X-ray crystallography structure of MMP9 in PDB provided favorable conditions for the study.

Matrix metalloproteinase 9 (MMP9), a member of the gelatinase family of matrix metalloproteinases (MMPs) that degrades ECM proteins, plays a major role in microvascular remodeling and cell migration during morphogenesis and wound healing (22-24). The expression level of MMP9 can be abnormally elevated in most tumors, and is subject to complex regulation. This could include a very complex regulation process at the level of transcription, mRNA dendritic translocation, and local translation as well as protein activation (23). Indeed, a high MMP9 level is associated with a poor prognosis in cancer patients (25-27).

The invasive nature and migratory capacity of tumor cells can be reduced by MMP inhibitors (28). The search for MMP inhibitors which are specifically designed to be safe and effective remains a 'hotspot' of cancer research. MMP9, an extracellular acting $\mathrm{Zn}^{2+}$-dependent endopeptidase, is released extracellularly in a latent, proform with the enzymatic site covered by a propeptide which needs to be severed off to expose the $\mathrm{Zn}^{2+}$ binding region, interact with the substrate and reveal the activity. From the model of interaction with the substrate or inhibitor, it can be divided into two parts: i) $\mathrm{Zn}^{2+}$ binding site of the catalytic activity center; ii) hydrophobic zone S2, S1', S1', S2' (29). As these active sites are important in enzymatic activity; therefore, targeting it may block enzymatic activity.

Computational techniques allow the evaluation of the binding affinity of compounds before synthesizing them in the laboratory (30). These computational techniques provide information concerning cancer-related genes. They, therefore, aid in the development of new inhibitory compounds.
Molecular docking is such a technique. Molecular docking is used in binding orientation of small molecules against their targets. This technique is also vital in the identification of new inhibitory compounds against certain diseases (31). This study focused on the design and molecular docking of cyclic peptides against active MMP9.

We studied the prospective for six peptides against active MMP9. The six peptides were stabilized, by adding a disulfide bridge, which converted them into the much more stable cyclic forms (32).

In the present study, peptides were docked with active MMP9 to determine their affinity as MMP9 inhibitors. Only top conformations were selected. The negative and low score for any ligand shows favorable interactions between the ligand and the receptor protein. Our results demonstrated that peptide 2 had a minimum $\mathrm{S}$ score and also had promising interactions with active site residues. All the peptides had low energy; they therefore formed a stable structure.

As observed in the study, active MMP9 was blocked by peptide 2. Thus, it may serve as a drug candidate for active MMP9. Additionally, peptide 5 and 6 had potential results that could serve as important drug candidates to block MMP9 activity. However, the designed cyclic peptides were not tested in mantle cell lymphoma in vivo nor in vitro. Further studies need to be conducted on the validity, metabolism, and side effects of the proposed peptides.

No drug has yet been developed to cure MCL, a rare and aggressive disease. New and better strategies are required to develop drug candidates that can treat MCL. This study is based on bioinformatic analysis of the MCL mechanism, and focuses on drug candidates that can target MMP9. MMPs are inhibited by compounds containing zinc-chelating groups, but few inhibitors specific for MMP9 have been developed; the most promising MMP9 inhibitors also inhibit other members of the MMP family (33). We used molecular docking to design selective MMP9 inhibitors based on protein structure. This study revealed cyclic peptide (CTFKEIVPDLC), which is able to interact with the sites of active MMP9.

The present study was proven to be valuable prior to synthesizing drugs. Additionally, cyclic peptides could be used as future candidates for drugs against MCL, as they possess binding affinity against MMP9. The results of this study are useful for drug development. The results can assist in the aided-screening of drugs against MCL.

\section{Acknowledgements}

Not applicable.

\section{Funding}

No funding was received.

\section{Availability of data and materials}

The datasets (GSE32018 and GSE9327) (11,12) analyzed during the current study are available in Gene Expression Omnibus (GEO) www.ncbi.nlm.nih.gov/pubmed.

Crystallographic data for MMP9 analyzed during the current study are available in Protein Data Bank (PDB) 
www.rcsb.org. The expression level of MMP9 analyzed during the current study is available in Oncomine www.oncomine. com (18). The immunohistochemistry staining results of MMP9 analyzed during the current study are available in the Human Protein Atlas database www.proteinatlas.org. The regulatory network and related transcription factors of MMP9 analyzed during the current study are available in Gene-RADAR www.gcbi.com.cn.

\section{Authors' contributions}

WY designed this study and conducted bioinformatic analysis and molecular docking. SXL participated in sorting the data and in drafting of the manuscript. HG participated in drafting of the manuscript, provided research guidance, and is the corresponding author. MW participated in drafting of the manuscript. All authors actively contributed to the paper on an intellectual level. All authors read and approved the final manuscript. All authors agreed to be accountable for all aspects of the work in ensuring that questions related to the accuracy or integrity of any part of the work are appropriately investigated and resolved.

\section{Ethics approval and consent to participate}

Not applicable.

\section{Patient consent for publication}

Not applicable.

\section{Competing interests}

The authors declare that they have no competing interests.

\section{References}

1. Li XY, Zhang L, Liu X, Feng L and Wang X: The antitumor effects of arsenic trioxide in mantle cell lymphoma via targeting Wnt $/ \beta$-catenin pathway and DNA methyltransferase-1. Oncol Rep 38: 3114-3120, 2017.

2. Cohen JB, Han X, Jemal A, Ward EM and Flowers CR: Deferred therapy is associated with improved overall survival in patients with newly diagnosed mantle cell lymphoma. Cancer 122 2356-2363, 2016.

3. Ratsch BA, Grau M, Döken B, Lenz P and Lenz G: The use of microarry technologies in mantle cell lymphoma. Semin Hematol 48: 166-171, 2011.

4. Fan F, Lu J, Yu W, Zhang Y, Su S, Pang L and Zhu B: MicroRNA-26b-5p regulates cell proliferation, invasion and metastasis in human intrahepatic cholangiocarcinoma by targeting S100A7. Oncol Lett 15: 386-392, 2018.

5. Klassen LMB, Chequin A, Manica GC, Biembengut IV, Toledo MB, Baura VA, de O Pedrosa F, Ramos EAS, Costa FF, de Souza EM, et al: $M M P 9$ gene expression regulation by intragenic epigenetic modifications in breast cancer. Gene 642: 461-466, 2018.

6. Yang XZ, Cui SZ, Zeng LS, Cheng TT, Li XX, Chi J, Wang R Zheng XF and Wang HY: Overexpression of Rab 1B and MMP9 predicts poor survival and good response to chemotherapy in patients with colorectal cancer. Aging 9: 914-931, 2017.

7. EI-Sharkawi F, EI Sabah M, Hassan Z and Khaled H: The biochemical value of urinary metalloproteinases 3 and 9 in diagnosis and prognosis of bladder cancer in Egypt. J Biomed Sci 21: 72, 2014.

8. Pouyanfar N, Monabbati A, Sharifi AA and Dianatpour M: Expression levels of MMP9 and PIWIL2 in prostate cancer: A case-control study. Clin Lab 62: 651-657, 2016.
9. Zhong Y, Lu YT, Sun Y, Shi ZH, Li NG, Tang YP and Duan JA: Recent opportunities in matrix metalloproteinase inhibitor drug design for cancer. Expert Opin Drug Discov 13: 75-87, 2018.

10. Kist R, Timmers LFSM and Caceres RA: Searching for potential mTOR inhibitors: Ligand-based drug design, docking and molecular dynamics studied of rapamycin binding site. J Mol Graph Model 80: 251-263, 2017.

11. Gómez-Abad C, Pisonero H, Blanco-Aparicio C, Roncador G, González-Menchén A, Martinez-Climent JA, Mata E, Rodríguez ME, Muñoz-González G, Sánchez-Beato M, et al: PIM2 inhibition as a rational therapeutic approach in B-cell lymphoma. Blood 118: 5517-5527, 2001.

12. Ruiz-Vela A, Aggarwal M, de la Cueva P, Treda C, Herreros B, Martín-Pérez D, Dominguez $\mathrm{O}$ and Piris MA: Lentiviral (HIV)-based RNA interference screen in human B-cell receptor regulatory networks reveals MCL1-induced oncogenic pathways. Blood 11: 1665-1676, 2008.

13. Liao YX, Zhang ZP, Zhao J and Liu JP: Effects of fibronectin 1 on cell proliferation, senescence and apoptosis of human glioma cells through the PI3K/AKT signaling pathway. Cell Physiol Biochem 48: 1382-1396, 2018.

14. Tan J, Qian X, Song B, An X, Cai T, Zuo Z, Ding D, Lu Y and Li H: Integrated bioinformatics analysis reveals that the expression of cathepsin $\mathrm{S}$ is associated with lymph node metastasis and poor prognosis in papillary thyroid cancer. Oncol Rep 40: 111-122, 2018.

15. Zang Y, Gu L, Zhang Y, Wang Y and Xue F: Identification of key genes and pathways in uterine leiomyosarcoma through bioinformatics analysis. Oncol Lett 15: 9361-9368, 2018.

16. Elkins PA, Ho YS, Smith WW, Janson CA, D'Alessio KJ, McQueney MS, Cummings MD and Romanic AM: Structure of the C-terminally truncated human Pro MMP9, a gelatin-binding matrix metalloproteinase. Acta Crystallogr D Biol Crystallogr 58: 1182-1192, 2002.

17. Appleby TC, Greenstein AE, Hung M, Liclican A, Velasquez M, Villaseñor AG, Wang R, Wong MH, Liu X, Papalia GA, et al: Biochemical characterization and structure determination of a potent, selective antibody inhibitor of human MMP9. J Biol Chem 292: 6810-6820, 2017.

18. Tian Y, Xu L, He Y, Xu X, Li K, Ma Y, Gao Y, Wei D and Wei L: Knockdown of RAC1 and VASP gene expression inhibits breast cancer cell migration. Oncol Lett 16: 2151-2160, 2018

19. Brune V, Tiacci E, Pfeil I, Döring C, Eckerle S, van Noesel CJ, Klapper W, Falini B, von Heydebreck A, Metzler D, Bräuninger A, et al: Origin and pathogenesis of nodular lymphocyte-predominant Hodgkin lymphoma as revealed by global gene expression analysis. J Exp Med 205: 2251-2268, 2008.

20. Steiner RE, Romaguera J and Wang M: Current trials for frontline therapy of mantle cell lymphoma. J Hematol Oncol 11: 13, 2018.

21. Martin P: Optimizing therapy for mantle cell lymphoma. Hematology Am Soc Hematol Educ Program 2017: 304-309, 2017.

22. Hou C, Miao Y, Ji H, Wang S, Liang G, Zhang Z and Hong W: 6-Gingerol inhibits hair cycle via induction of MMP2 and MMP9 expression. An Acad Bras Cienc 89: 2707-2717, 2017.

23. Phillips TM, Fadia M, Lea-Henry TN, Smiles J, Walters GD and Jiang SH: MMP2 and MMP9 associate with crescentic glomerulonephritis. Clin Kidney J 10: 215-220, 2017.

24. Sakata K, Satoh M, Someya M, Asanuma H, Nagakura H, Oouchi A, Nakata K, Kogawa K, Koito K, Hareyama M and Himi T: Expression of matrix metalloproteinase 9 is a prognostic factor in patients with non-Hodgkin lymphoma. Cancer 100: 356-365, 2004

25. Xue Q, Cao L, Chen XY, Zhao J, Gao L, Li SZ and Fei Z: High expression of MMP9 in glioma affects cell proliferation and is associated with patient survival rates. Oncol Lett 13: 1325-1330, 2017.

26. Klimczak-Bitner AA, Kordek R, Bitner J, Musial J and Szemraj J: Expression of MMP9, SERPINE1 and miR-134 as prognostic factors in esophageal cancer. Oncol Lett 12: 4133-4138, 2016.

27. Yu Y, Ding Z, Jian H, Shen L, Zhu L and Lu S: Prognostic value of MMP9 activity level in resected stage I B lung adenocarcinoma. Cancer Med 5: 2323-31. 2016.

28. Piperigkou Z, Manou D, Karamanou K and Theocharis AD: Strategies to target matrix metalloproteinases as therapeutic approach in cancer. Methods Mol Biol 1731: 325-348, 2018.

29. Vandooren J, Van den Steen PE and Opdenakker G: Biochemistry and molecular biology of gelatinase B or matrix metalloproteinase-9 (MMP-9): The next decade. Crit Rev Biochem Mol Biol 48: 222-272, 2013. 
30. Khan MF, Nahar N, Rashid RB, Chowdhury A and Rashid MA Computational investigations of physicochemical, pharmacokinetic, toxicological properties and molecular docking of betulinic acid, a constituent of Corypha taliera (Roxb.) with Phospholipase A2 (PLA2). BMC Complement Altern Med 18: 48, 2018.

31. Kumar A, Srivastava G, Negi AS and Sharma A: Docking, molecular dynamics, binding energy-MM-PBSA studies of naphthofuran derivatives to identify potential dual inhibitors against BACE-1 and GSK-3ß. J Biomol Struct Dyn 19: 1-16, 2018.

32. Ojo OS, Nardone B, Musolino SF, Neal AR, Wilson L, Lebl T, Slawin AMZ, Cordes DB, Taylor JE, Naismith JH, et al: Synthesis of the natural product descurainolide and cyclic peptides from lignin-derived aromatics. Org Biomol Chem 16: 266-273, 2018.
33. Gossage DL, Cieslarová B, Ap S, Zheng H, Xin Y, Lai P, Chen G, Smith V and Sundy JS: Phase 1b study of the safety, pharmacokinetics, and disease-related outcomes of the matrix metalloproteinase-9 inhibitor andecaliximab in patients with rheumatoid arthritis. Clin Ther 40: 156-165, 2018.

(i) (9) This work is licensed under a Creative Commons EY No No Attribution-NonCommercial-NoDerivatives 4.0 International (CC BY-NC-ND 4.0) License. 